\title{
Licht in Musik - Wort - Bild
}

David Künzler

Dr. med. David Künzler Jakob Zürrerstrasse 35 CH-8915 Hausen am Albis

dkkuenzler@bluewin.ch

www.aerzte-kunst.ch

www.asem-vssa.ch

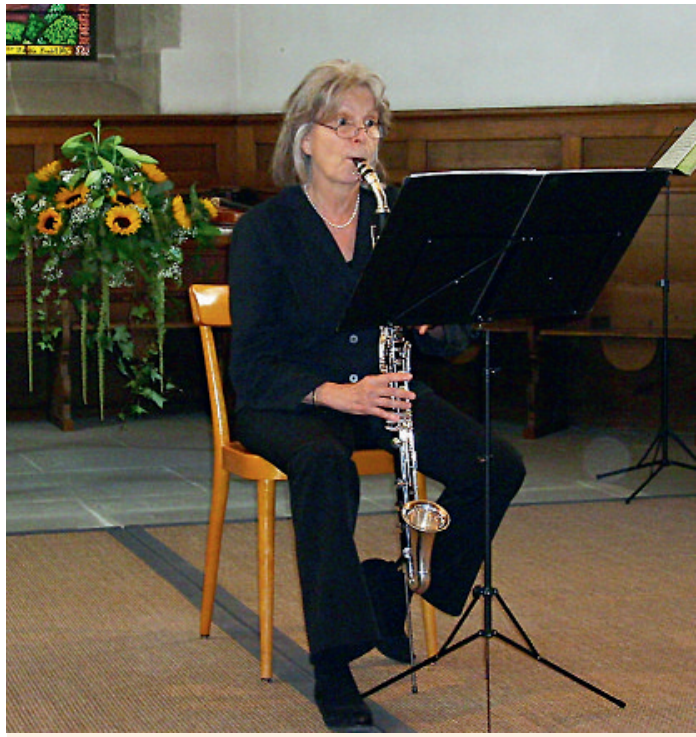

Elisabeth Darbellay spielt Bassetthorn.

Ein Konzert in der Französischen Kirche in Murten, dargeboten vom Musikerarzt Dr. Jean-Luc Darbellay und Mitgliedern seiner Familie sowie eine Lesung aus Werken von Schriftstellerärzten bildeten den Anfang der Ausstellungseröffnung mit Werken der Vereinigung malender und plastisch tätiger Ärztinnen und Ärzte. Sie fand vom 11. Juli bis zum 2. August 2009 im Rathauskeller in Murten statt.

Claude Debussy soll über Richard Strauss gesagt haben: «Bei ihm ist alles Licht.» So kam es, dass wir das Thema «Licht» wählten, ausgedrückt in Musik, Wort und Bild.

\section{Das Konzert}

Lichtvoll, man kann auch sagen strahlend, waren die Werke von Bach, Sierra, Darbellay, Bartók, Haydn und Beethoven. J.-L. Darbellay meinte auf die Frage, welches Werk am meisten Licht in sich hätte, alles sei voll Licht, «ohne Licht keine Musik».

Es war ein Vergnügen zuzuhören, wie N.-A. Darbellay (Violine) und F. Sierra (Viola) zu Beginn drei Duette von Johann Sebastian Bach erklingen liessen, gefolgt von einer Komposition und Dichtung von F. Sierra, mit klarer Stimme und Violine vorgetragen von N.-A. Darbellay. E. Darbellay brachte dann in «Espace für Bassetthorn» (von J.-L. Darbellay) die warmen, ruhig klingenden Töne des wenig bekannten Instruments zu Gehör (s. Abb. oben). So leuchtete denn durch alle gespielten Werke Helligkeit, auch beim Trio für Violine, Klarinette und Bassetthorn mit J.-L. Darbellay an der Klarinette. Die Musiker, die in den vergangenen Wochen weit gereist waren (u.a. Auftritte in Tokio) ernteten grossen Beifall. Dankbar wurde anerkannt, dass sie im kleinen Murten für uns da waren.

\section{Die Lesung}

Csilla Bekes erzählte in dicht hintereinander gedrängten Sätzen, wie ihr und ihrer Schwester in der Kindheit im wahrsten Sinn die Augen geöffnet wurden für Verfehlungen der Eltern, geschehen im Dunkeln, grell für Sekunden beleuchtet durch ungewollt eingeschaltetes Licht. Ein bleibendes Trauma. «Das Licht war schuld.»

Hansruedi Gehring nahm uns mit in einen Traum, der ihm beim nachträglichen Analysieren eine momentane Lebenssituation erhellte und half, in einer verzwickten Situation mit einem Kollegen den weiteren Weg zu finden.

Helga Thomas las Gedichte aus ihrer Sammlung «Lichträume». Susi Dennler ging ein auf den Bildhauer Vincenzo Vela und sein in Airolo stehendes Relief zu Ehren der beim Tunnelbau verunfallten und gestorbenen Arbeiter. Als Gipsrelief 1882 zur Einweihung des Tunnels geschaffen, wurde es an der Landesausstellung 1883 gezeigt, aber erst 1932 zur 50-Jahr-Feier in Bronze gegossen. Sie schilderte auch den Hintergrund der sogenannten Minenkrankheit. Dennler liess sich vom Relief zu Ölbildern inspirieren, welche die Leiden der Arbeiter, aber auch Hoffnung ausdrücken (s. Bild unten).

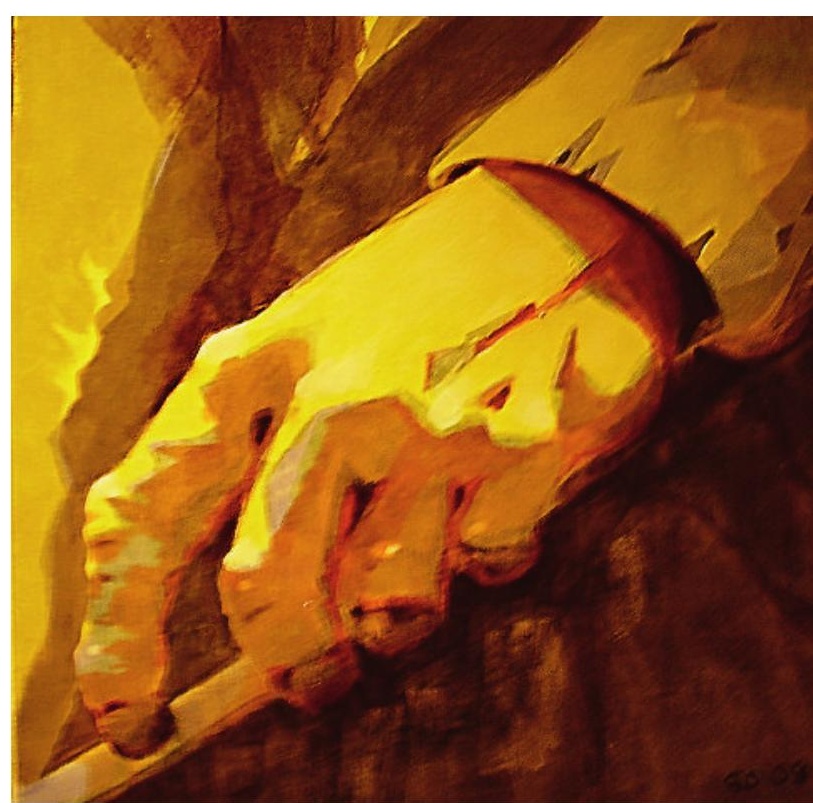

Am Ende des Tunnels - Ölbild von Susi Dennler. 


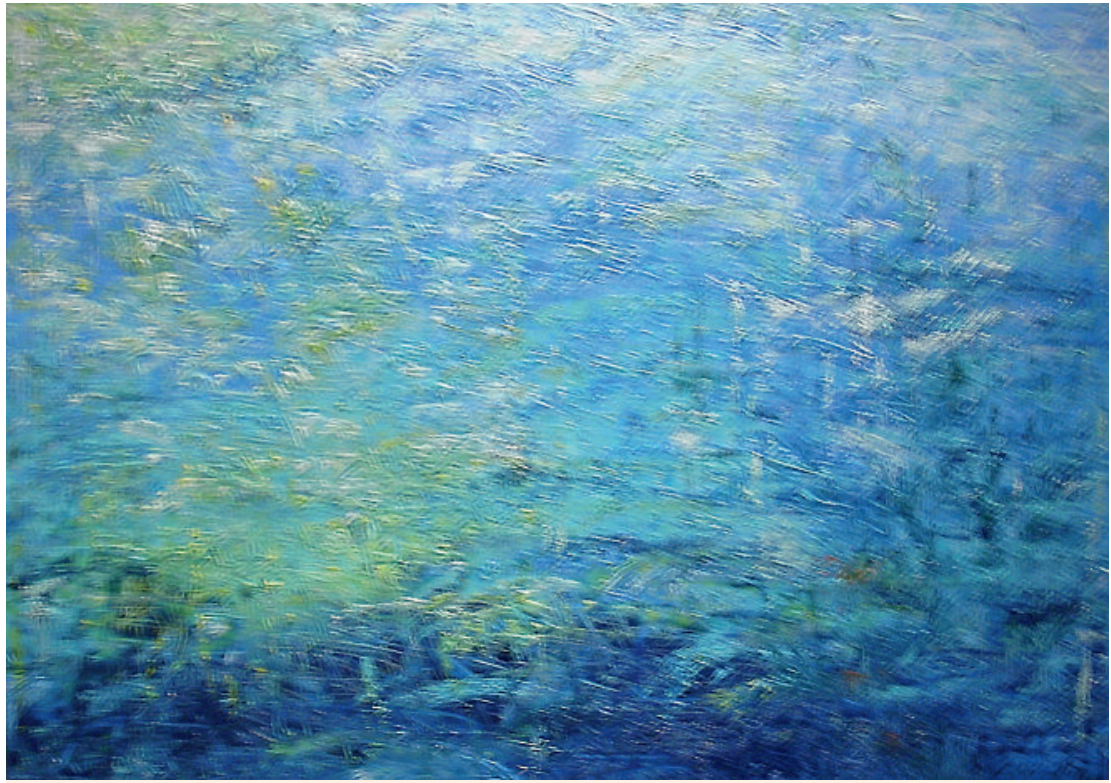

Lichtreflexe unter Wasser - ein Bild in Wasser-Acryl-Mischtechnik von Harriet Keller.

Bei Harriet Kellers Text «Verlorene Bahn» leuchtet zu Beginn sanft, golden und warm die Herbstsonne. Doch - unerklärt - kamen Schatten über die Sonne, es brauste und wurde Nacht, Angst ging um, die Wahrnehmung der Menschen engte sich ein, und «eine beschleunigende Kraft drehte die Welt unaufhaltsam. Die Erde rotierte schneller und schneller. Sie verlor ihre Bahn (...) und alle Wesen waren verloren.» Im Gegensatz zu diesem hoffnungslosen Geschehen malte Keller ein in der Tiefe und an der Oberfläche leuchtendes Bild für die Ausstellung (s. Bild oben).

Dietmar Thumm liess sich wortgestalterisch von grossen Dichtern inspirieren: «Variationen von Lichtern - nach berühmten Dichtern»:

Das Licht (nach C. F. Meyer)

Herrlich strahlend auf der Erde Glanz

Ergiesst sich das Licht vom Blau

Des Himmels Rund und gibt sich ganz

Der Farbe hin und auch des Wassers Tau.

So glänzet alles weiss und hell,

glitzert und spiegelt, bricht das Licht, und wo es leuchtet farbig und grell des Schattens Dunkelheit gebricht.

Der Geistesblitz (nach Wilhelm Busch) Packt dich mit lautem Donnergrollen Ein gar zu heftig inn'res Wollen, Begeistern dich solche Gedanken, Dass sprengen sie gleich alle Schranken, Sogar die Idee dich ganz erhitz dann redet man vom Geistesblitz.
Man sollte niemand dafür schelten Jedoch, er ist bei vielen Menschen selten. Drum gelten scheele Blicke jenen, Die ihn haben, denn bei denen Leuchtet im Kopf der Kandelaber. Gerade drum, oder auch aber Generell ist man der Meinung das sei nur eine Lichterscheinung.

Die Bilderausstellung bereicherte Thumm mit ganz feinen, sozusagen durchscheinenden Zeichnungen.

\section{Die Bilderausstellung}

24 Mitglieder der Vereinigung stellten über 100 Werke aus. Es war eine helle Schau, bei der aber die Schatten naturgemäss nicht fehlten. Wie gewohnt zeigte sich eine grosse Vielfalt der Darstellungen, Stile und Materialien, Arbeiten in Acryl, Pastellkreide, Aquarell, Öl, Mischtechniken wurden gezeigt. Ein Beispiel ist das Bild in Acryl-Kreide-Mischtechnik von Christa Ritter (s. unten).

Zum Abschluss zwei Zitate aus dem Besucherbuch: «Viele schöne Bilder, berührend, professionell ausgeführt, grossartig.» «Spannend, wie unterschiedlich Empfindungen ausgedrückt und wiedergegeben werden.»

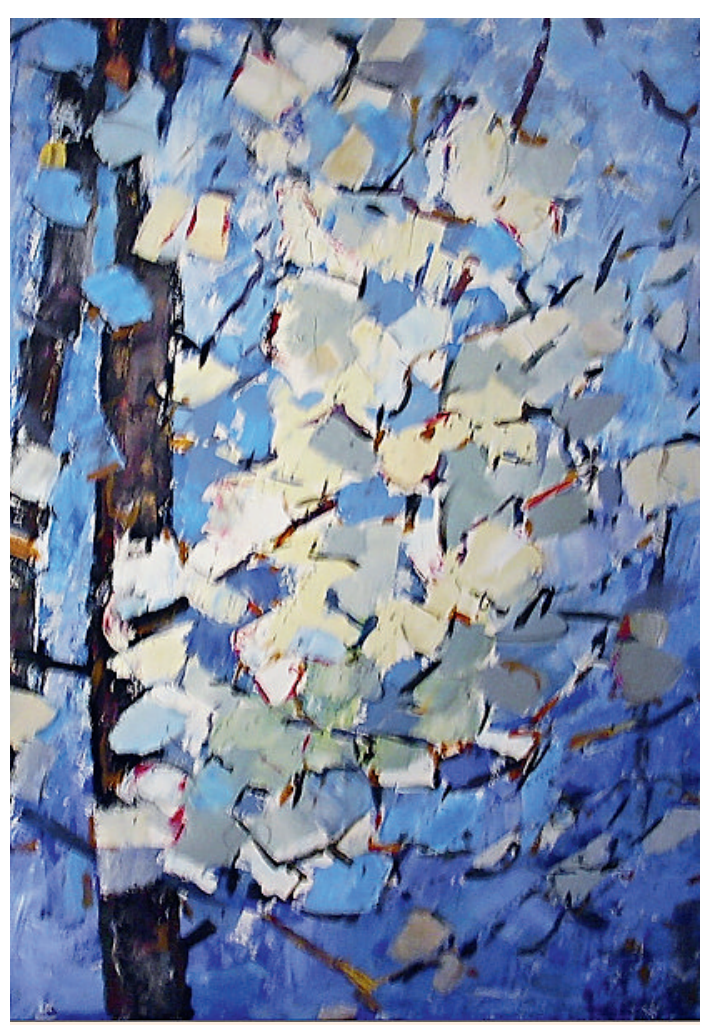

Magnolien - von Christa Ritter in Acryl-KreideMischtechnik gemalt. 